\title{
Dinoflagellés toxiques et phénomènes d'eaux colorées sur les côtes françaises : évolution et tendances entre 1975 et 1988.
}

\author{
C. Belin, J.P. Berthome, P. Lassus \\ IFREMER - Route de I'lle d'Yeu - B.P. 1049. 44037 Nantes Cedex 01.
}

\section{1 - POSITION DU PROBLEME}

Très peu de laboratoires européens disposent actuellement de données hydrologiques et biologiques à long terme, soit sur plusieurs dizaines d'années, en ce qui concerne l'évolution des populations phytoplanctoniques sur une zone pilote. On doit néanmoins citer les résultats obtenus en Mer du Nord et dans l'Atlantique Nord par le «Continuous Plankton Recorder Survey» (GLOVER, 1967, COLEBROOK et al., 1984), en German Bight par le laboratoire d'Helgoland (RADACH et BERG, 1986), en Mer Adriatique par le centre de recherche marine de Rovinj - Yougoslavie (SMODLAKA et REVELANTE) et en Mer du Nord par le MBA de Plymouth (MASSOCK et al., 1981). Le degré d'eutrophisation croissant de la plupart des zones côtières nord européennes rend aujourd'hui nécessaire une estimation réaliste d'une transformation éventuelle des écosystèmes phytoplanctoniques dans ces zones. A ce titre les études de tendance sont souhaitables, non seulement pour contrôler l'ensemble des successions végétales saisonnières, mais également pour suivre la séquence d'apparition des blooms exceptionnels, qu'il s'agisse de phénomènes d'eaux colorées ou de manifestations épisodiques d'espèces à caractère toxique. Un recensement a été accompli sur les côtes françaises depuis 1978 et quelques données sont disponibles depuis 1975. L'ensemble des observations recueillies en 13 ans peut donc être utilisé dans le but de détecter une augmentation anormale de l'un et l'autre de ces phénomènes.

On peut dire que les phénomènes d'eaux colorées ont peu intéressé l'opinion publique française, hormis quelques scientifiques, jusqu'en 1978. En effet, cette année là, une eau rouge de grande ampleur $(30 \mathrm{~km})$ pouvait être observée sur la côte normande pendant deux semaines. Les organismes responsables: Gonyaulax 
polygramma et G. spinifera étaient rapidement identifiés, et, vu le caractère non toxique de ces deux dinoflagellés, tout risque vis-à-vis de la conchyliculture locale a été écarté (LASSUS et al., 1980). Néanmoins, cette manifestation survenant quelques mois après la catastrophe de l'«Amoco-Cadiz" sur les côtes bretonnes, chacun a voulu établir une relation entre «marée rouge" et niveau de pollution des baies et des estuaires, même si cette hypothèse a été infirmée dans ce cas (LE FEVRE, 1979).

II n'en demeure pas moins que la sensibilisation croissante de l'opinion publique depuis 1978 a permis d'augmenter les informations disponibles aussi bien sur les colorations des eaux de surface en été que sur les mortalités d'animaux marins liées à la présence de certains microorganismes phytoplanctoniques, ou encore sur les intoxications alimentaires humaines.

De fait, si la ressource conchylicole française semble n'avoir jamais été affectée par des empoisonnements du type P.S.P. (1), (MARTEIL et PAULMIER, 1970) un réseau de surveillance mis en place depuis 1981 par I'Institut Scientifique et Technique des Pêches Maritimes (I.S.T.P.M.) a tenté de prévoir de tels phénomènes. En effet, en 1900 et 1905, des intoxications paralytiques (Mytilitoxisme) avaient été signalées dans le nord de la France, respectivement à Boulogne et au Havre, avec 4 décès observés dans le premier cas et 3 dans le second. De plus, en 1976, 33 intoxications PSP dues à des moules

(1) PSP: Paralytic Shellfish Poison. importées d'Espagne ont été recensées, Cependant, l'absence quasi providentielle de ce type d'intoxication sur les côtes françaises entre 1900 et aujourd'hui, alors que de nombreux pays européens étaient touchés par le phénomène dès les années 60 , a permis de penser que les organismes responsables (Protogonyaulax tamarensis par exemple) étaient absents de nos côtes. II a fallu attendre l'été 1988 pour constater le contraire puisque des contaminations de moules et huîtres en Bretagne nord étaient dues à Alexandrium minutum, espèce considérée jusqu'en 1987 (HAELLEGRAEF et al., 1988) comme non productrice de PSP.

Par ailleurs, plus de 3000 intoxications diarrhéiques provoquées en 1983 par la présence simultannée de plusieurs espèces de Dinophysis en Bretagne sud et sur la côte normande, ont rapidement fait prendre conscience de l'importance du problème économique (interdictions de commercialisation des coquillages) posé par les manifestations de dinoflagellés toxiques.

Enfin, les eaux colorées à Gyrodinium aureolum observées périodiquement en Bretagne depuis 1978 et associées à des effets létaux ou sublétaux sur poissons et coquillages ont nécessité une attention croissante des services publiques.

Ceci s'est concrétisé en 1984 par la mise en place à IFREMER ${ }^{(2)}$ d'un nouveau système de surveillance reposant à la fois sur des examens phy-

(2) Institut Français de Recherche et d'Exploitation de la Mer. 
toplanctoniques et sur des tests de toxicité $\mathrm{DSP}^{(3)}$ ou PSP, ce second niveau de contrôle étant conditionné par le premier. C'est grâce à ce réseau qu'un maximum d'information a été recueilli dès 1984 aussi bien sur les espèces toxiques que sur celles provoquant des eaux rouges non toxiques en plus de 60 points de prélèvements répartis sur tout le littoral.

\section{2 - EAUX COLOREES A CARACTERE NON TOXIQUE}

Afin de mieux apprécier l'évolution des eaux colorées au cours des 13 dernières années, nous distinguerons respectivement: 1) les diatomées, 2) les dinoflagellés, 3) les autres flagellés (Haptophycées, Chlorophycées, Cryptophycées) et les ciliés.

La figure 1 montre l'évolution du nombre de blooms planctoniques observés chaque année depuis 1975. Le nombre total des eaux colorées liées aux différents groupes est souvent supérieur au nombre total de perturbations signalées en raison de l'existence d'eaux colorées plurispécifiques.

L'année 1978 a été l'une des plus fertiles en phénomènes de ce type. Au cours de cette année, en raison de la catastrophe de l'Amoco-Cadiz,

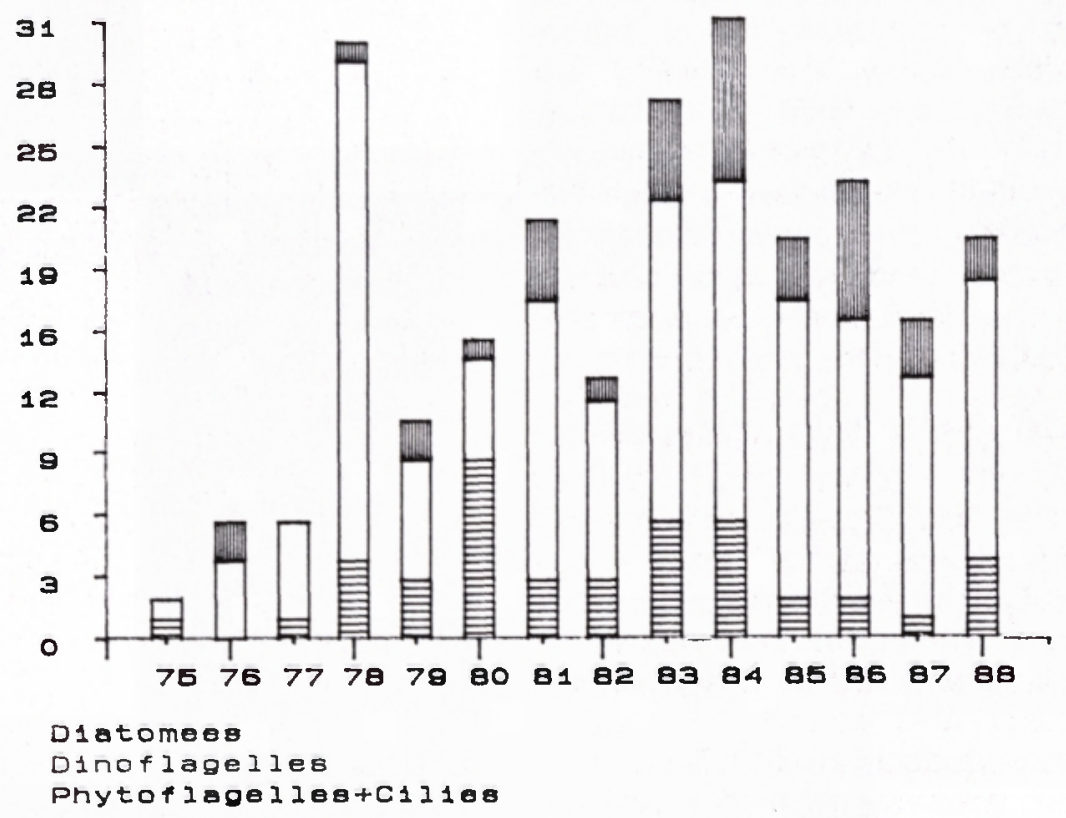

Fig. 1. - Nombres cumulés de cas d'eaux colorées sur les côtes françaises entre 1975 et 1988 , pour les diatomées, les dinoflagellés et les phytoflagellés. Occurrences of discolored waters along french coasts between 1975 and 1988 : compiled number of cases for diatoms, dinoflagellates and phytoflagellates.

(3) Diarrheic Shellijsh Poison. 
nombreux ont été ceux qui ont voulu établir une relation entre les apparitions d'eaux colorées à dinoflagellés et le niveau de pollution des estuaires ou des baies.

Ces hypothèses ont été infirmées dans ce cas de pollution massive par des hydrocarbures (LE FEVRE, 1980). Si l'on ne tient pas compte de cette année exceptionnelle, depuis 1980 , la tendance générale est à l'augmentation de ces phénomènes. On peut cependant penser que la mise en place de réseaux de surveillance amplifie cette tendance (plus grande proportion de phénomènes déclarés et observés par rapport au nombre réel d'apparitions d'eaux colorées). Enfin, depuis 1984, le nombre total d'eaux colorées par an semble en diminution avec un niveau annuel moyen compris entre 15 et 20 cas, et ceci jusqu'en 1988. La dominance par classe phytologique est quasi-invariable: chaque année dinoflagellés et diatomées représentent l'essentiel des cas signalés par rapport aux phytoflagellés + ciliés.

Certains secteurs sont plus touchés que d'autres, il s'agit en particulier :

* des estuaires

* des bassins semi-fermés tels que le golfe d'Arcachon, les baies de Vilaine, de Quiberon et de Douarnenez

* des enceintes portuaires

* des discontinuités des masses d'eau au niveau du talus continental (Mer d'Iroise, Ouessant, Manche Ouest) : dans ce dernier cas, les seules espèces répertoriées sont soit Noctiluca scintillans, soit Gyro- dinium aureolum ou plus rarement Emiliana huxleyi

Nous avons présenté sur la figure 2 les zones les plus représentatives des côtes françaises en ce qui concerne les manifestations d'eaux colorées. II est intéressant de souligner que les secteurs côtiers où les fréquences d'apparition estivale d'eaux colorées sont les plus fortes correspondent à des zones plus ou moins industrialisées mais surtout proches du débouché d'un grand fleuve (Seine, Loire) où d'une rivière draînant un important bassin versant (Vilaine).

De 1975 à 1984, chez les diatomées, Rhyzosolenia delicatula $(7 \%$ des cas) et Nitzschia seriata $(1,5 \%)$ représentent à elles seules la moitié des eaux colorées à diatomées (14 espèces déterminées) et pendant la même période par rapport à l'ensemble des observations d'eaux colorées, trois espèces de dinoflagellés non toxiques dominent:

Noctiluca scintillans $23 \%$ des cas Prorocentrum micans $13 \%$ Gonyaulax sp. $\quad 11 \%$

Enfin, les eaux colorées à ciliés sont presque exclusivement composées de Mesodinium rubrum, de même que les haptophycées par Phaeocystis pouchetii et les chlorophycées par Pyramimonas disomata et Nannochloris sp.

Nous avons donc porté plus particulièrement notre attention sur les dinoflagellés, phytoflagellés et ciliés les plus abondants dans les manifestations d'eaux colorées pendant ces 13 dernières années. Ces résultats sont résumés dans le tableau 1 d'après 


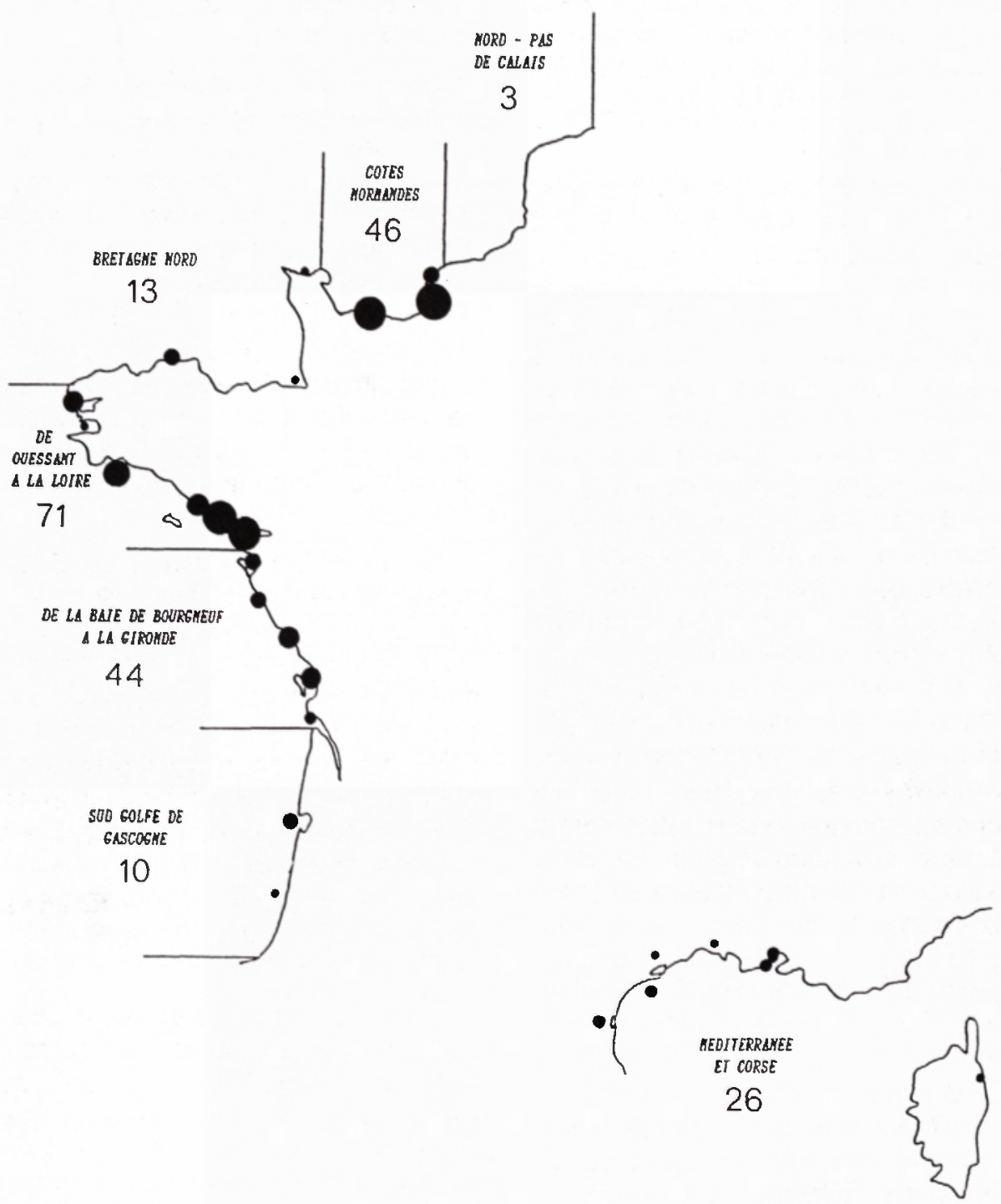

Fig. 2. - Nombres totaux d'eaux colorées pour chaque zone géographique de surveillance conchylicole et classification : le diamètre des cercles est proportionnel au nombre d'eaux colorées recensées par zone entre 1975 et 1988. Total numbers of discolored waters for each main areas of shellfish monitoring and classification of each coastal area subjected to sampling operations : diameter of black circles are proportional to the number of discolored waters between 1975 and 1988. 
Table I. Nombre de cas d'eaux colorées de 1975 à 1988 pour les espèces dominantes lors des blooms. (") données recueillies à partir d'observations ponctuelles et à partir d'un suivi permanent des eaux au large de Gravelines (Mer du Nord). Number of cases of discolored waters from 1975 to 1988 and for the main blooming species. (*) data compiled from observed discolored water and from a continuous survey of offshore waters at Gravelines (North Sea).

\begin{tabular}{|c|c|c|c|c|c|c|c|c|c|c|c|c|c|c|c|}
\hline Espèces & 1975 & 1976 & 1977 & 1978 & 1979 & 1980 & 1981 & 1982 & 1983 & 1984 & 1985 & 1986 & 1987 & 1988 & Total \\
\hline $\begin{array}{l}\text { Dinoflagellés } \\
\text { Prorocentrum micans } \\
\text { Procorentrum minimum } \\
\text { Noctiluca scintillans } \\
\text { Gymnodinium aureolum } \\
\text { Gonyaulax spp. } \\
\text { Gymnodinium sp. } \\
\text { (couleur verte) }\end{array}$ & $\begin{array}{l}1 \\
- \\
- \\
- \\
- \\
-\end{array}$ & $\begin{array}{l}2 \\
- \\
1 \\
1 \\
- \\
-\end{array}$ & $\begin{array}{l}2 \\
- \\
- \\
-\end{array}$ & $\begin{array}{l}3 \\
- \\
9 \\
3 \\
6 \\
-\end{array}$ & $\begin{array}{l}- \\
- \\
2 \\
- \\
1 \\
-\end{array}$ & $\begin{array}{l}2 \\
- \\
- \\
- \\
- \\
-\end{array}$ & $\begin{array}{l}5 \\
1 \\
5 \\
2 \\
1 \\
-\end{array}$ & $\begin{array}{l}2 \\
1 \\
1 \\
- \\
- \\
1\end{array}$ & $\begin{array}{l}1 \\
1 \\
3 \\
2 \\
2 \\
3\end{array}$ & $\begin{array}{l}1 \\
2 \\
9 \\
- \\
4 \\
-\end{array}$ & $\begin{array}{l}2 \\
\overline{7} \\
- \\
1 \\
3\end{array}$ & $\begin{array}{l}- \\
7 \\
4 \\
1 \\
1 \\
1\end{array}$ & $\begin{array}{l}3 \\
6 \\
-6 \\
1 \\
2\end{array}$ & $\begin{array}{l}1 \\
2 \\
5 \\
1 \\
- \\
3\end{array}$ & $\begin{array}{l}25 \\
20 \\
46 \\
16 \\
18 \\
13\end{array}$ \\
\hline $\begin{array}{l}\text { Haplophycées } \\
\text { Phaeocystis pouchetii (*) }\end{array}$ & * & - & 2 & 2 & 2 & 4 & 2 & 2 & 2 & 2 & 2 & 1 & - & 1 & 23 \\
\hline $\begin{array}{l}\text { Ciliés } \\
\text { Mesodinium rubrum }\end{array}$ & 1 & - & - & - & - & 3 & 1 & 1 & 2 & 2 & 1 & - & - & 1 & 12 \\
\hline
\end{tabular}

plusieurs synthèses (BELIN et BERTHOME, 1988; BERTHOME et BELIN, 1988; BERTHOME et LASSUS, 1986; GROSSEL comm. pers.).

Bien que la Noctiluque, comme dans de nombreux pays, reste l'organisme d'eau rouge le plus cité, il est clair que cela ne ressort pas d'une quelconque action toxique mais bien de l'aspect particulier "rouge brique» des colorations estivales côtières dues à ce dinoflagellé de grande taille (100 à $500 \mu \mathrm{m})$. Les eaux colorées à Prorocentrum sont également bien représentées en zones estuariennes, de même que celles à Gonyaulax ( $G$. spinifera, $G$. digitalis,...) non toxiques. Ces espèces sont par ailleurs connues pour leur affinité pour les eaux dessalées. Néanmoins, depuis 1986, les apparitions de Prorocentrum minimum semblent plus fréquentes en baie de Seine et dans le Golfe du Lyon (Méditerranée) ${ }^{(1)}$. Les dévelop- pements importants de Gyrodinium aureolum (plus de $10^{6}$ cellules/litre) sont aléatoires et moins fréquents excepté en 1987 - que les blooms de moindre importance détectés en juinjuillet en Bretagne nord et sud.

Cette espèce n'est pas toxique pour l'homme et de ce fait, a été inclue dans cette liste. Elle sera néanmoins étudiée plus loin au titre des espèces toxiques pour la faune marine. Enfin, si les manifestations d'eaux ocres ou rouges dues respectivement à Phaeocystis pouchetii et Mesodinium rubrum sont assez fré-

(1) On ne peut pas clairement attribuer cet accroissement à un facteur de croissance présent dans le milieu. Néanmoins cette espèce semble sensible expérimentalement à un enrichissement en fer chélaté du milieu de culture et le fait que les zones où elle prolifère sont soumises à des apports industriels ou urbains riches en fer devrait orienter des recherches futures. 
quentes à irrégulières sur une décennie, il reste que depuis 1983 le littoral nord atlantique - particulièrement entre l'île d'Oléron et le nord de l'estuaire de la Loire - est de plus en plus touché par des "eaux vertes" constituées de densités énormes d'un petit Gymnodinium non identifié à ce jour. Ces développements tendent à être de plus en plus fréquents, bien qu'ils ne s'accompagnent d'aucun effet sur la faune ou sur le consommateur de coquillage. Malgré des plaintes récentes (1988) provenant de baigneurs ayant déclaré des maladies de peau à la suite d'un contact avec cette "marée verte", des tests allergologiques réalisés à Nantes (Laboratoire de cosmétologie et pharmacologie industrielle) se sont révélés négatifs.

\section{3 - DINOFLAGELLES TOXIQUES}

Trois genres de dinoflagellès font actuellement l'objet d'une attention particulière au niveau des programmes de surveillance et de recherche mis en place par IFREMER depuis 1984: Dinophysis, Gyrodinium et Alexandrium. Nous reprendrons la chronologie des observations réalisées pour chaque espèce concernée.

\section{a. Dinophysis}

Ce n'est qu'en 1983 que Dinophysis acuminata a été considérée comme

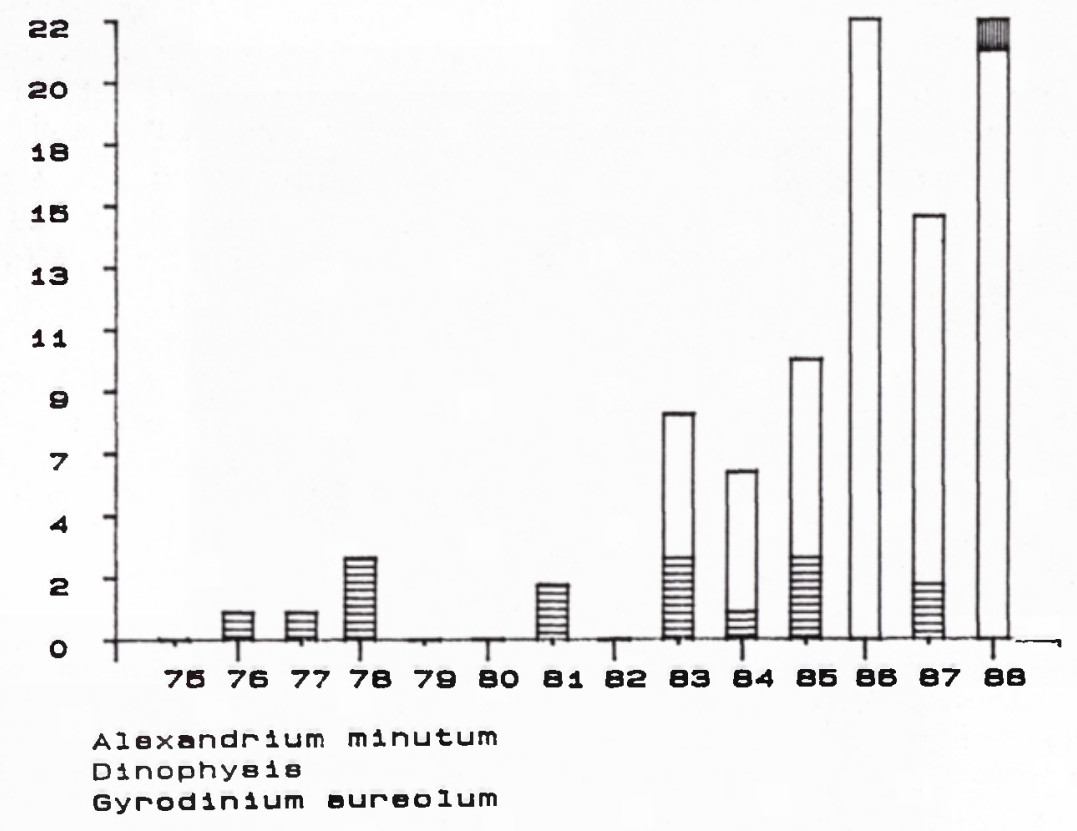

Fig. 3. - Nombres cumulés de blooms à A. minutum, Dinophysis spp. et G. aureolum. Compiled numbers of bloom events due to $A$. minutum, Dinophysis and $G$. aureolum. 

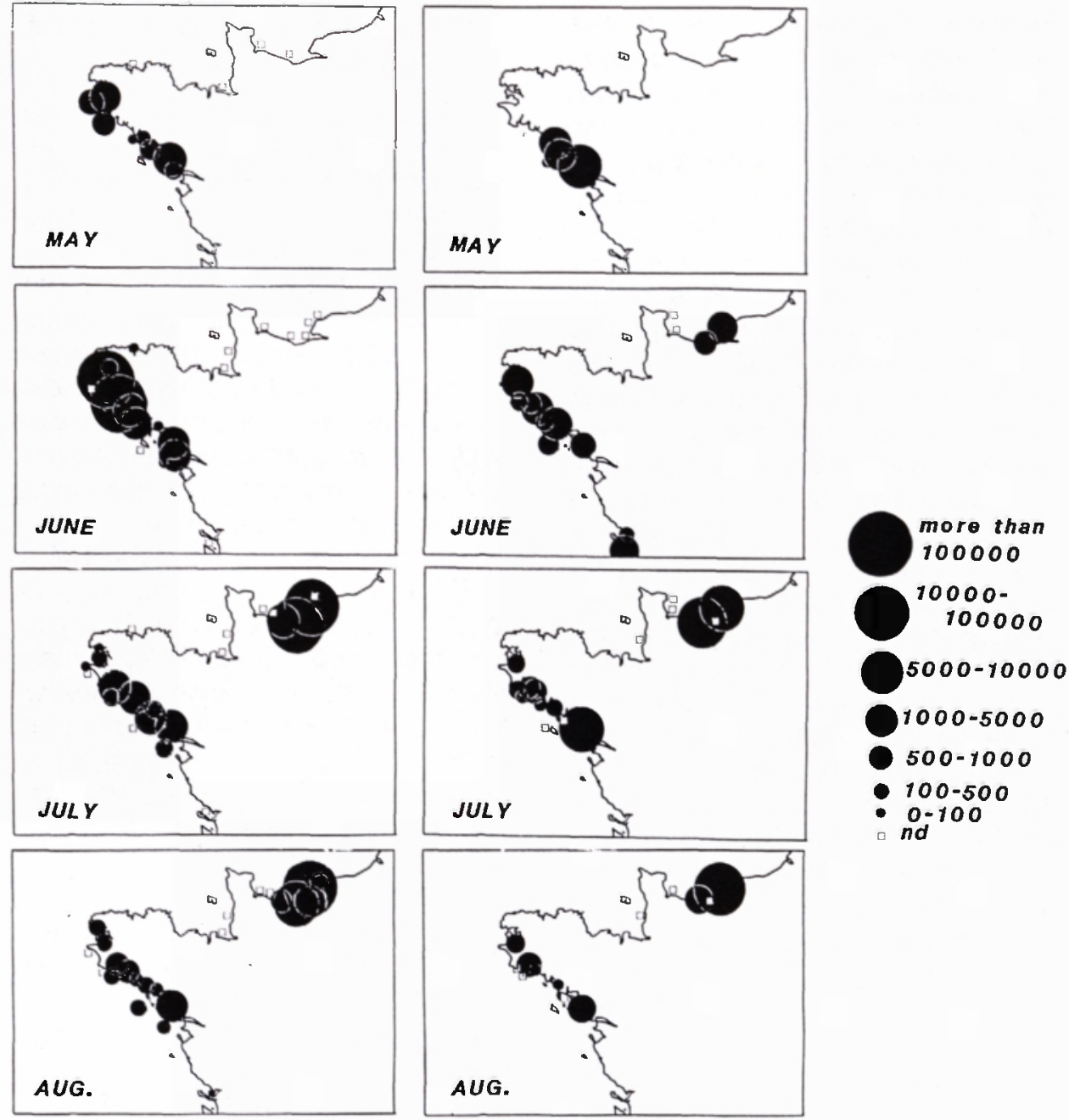

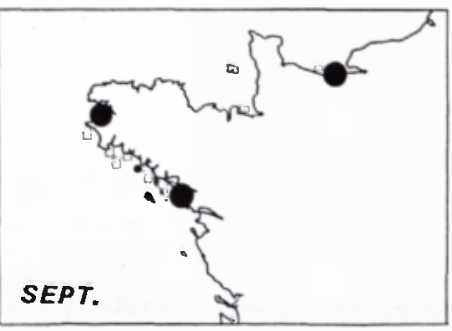

1986

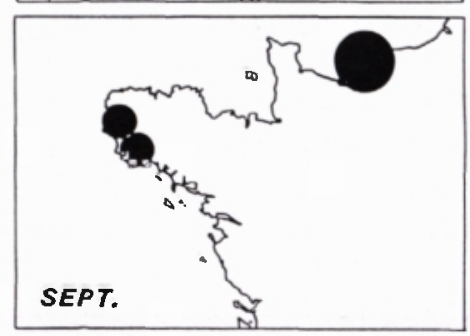

1987

Fig. 4. - Distribution saisonnière de Dinophysis spp le long des côtes de la Manche et de l'Atlantique en 1986 et 1987 (concentrations cellulaires de surface exprimées en cellules/dm ${ }^{3}$ ). Seasonal distribution of Dinophysis spp along Channel and Atlantic coasts in 1986 and 1987 (surface concentrations expressed as cell/dm3). 
l'espèce responsable des intoxications diarrhéiques observées chez les consommateurs de moules en Bretagne sud et en Normandie. Néanmoins, de telles intoxications, non attribuables à des gastro-entérites d'origine bactérienne, avaient également été signalées en 1978 et 1981 en baie de Vilaine, La Présence de Plusieurs espèces de Dinophysis $(D$. sacculus, $D$. acuminata, $D$. norvegica ?) en zone côtière n'est donc probablement pas un phénomène apparu en 1983 mais existant déjà de nombreuses années plutôt. De ce fait les estimations du nombre d'apparitions (fig. 3) sous-estiment certainement la réalité entre 1975 et 1983, II semble néanmoins que la surveillance exercée depuis 1983 démontre une certaine augmentation des blooms à Dinophysis de même qu'une extension vers des zones non contaminées en 1983 (Charentes-Vendée et littoral méditerranéen). La distribution côtière mensuelle dans les eaux de surface suit un scénario similaire chaque année entre mai et septembre (fig. 4): le maximum saisonnier a lieu en juin pour la Bretagne sud et en juillet pour la Normandie, soit respectivement pour $D$. sacculus et $D$. acuminata.

Sur le plan sanitaire, au cours des étés 1983 et 1984, de nombreuses intoxications à D.S.P, ont été signalées. II est vrai que les bilans globaux sont souvent difficiles à établir avec précision car de nombreux consommateurs atteints de gastro-entérites ne vont pas obligatoirement consulter un médecin ou même un pharmacien. De plus, en raison d'une symptomatologie proche, il peut y avoir confusion entre des gastroentérites d'origine microbienne (coquillières ou non) et de type D.S.P.

En 1983, pour le seul département de la Loire-Atlantique, 3300 cas ont été estimés (443 cas prouvés). Sur les côtes du Calvados, le nombre de déclarations d'intoxication a été voisin de 150 . En 1984, on peut dénombrer 70 cas d'intoxication en Loire-Atlantique avec des coquillages en provenance de la baie de Vilaine, 4 par des coquillages de la baie de Douarnenez, quelques dizaines par des coquillages provenant des côtes du Calvados, par contre, entre 1500 et 2000 personnes ont été intoxiquées par des moules pêchées sur le gisement de Barfleur. En effet, le temps nécessaire entre le résultat des tests "souris" et de la décision administrative est de l'ordre de 48 heures après le prélèvement. Durant ce laps de temps, entre 150 et 200 tonnes de moules ont èté pêchées et expédiées dans toute la France.

A part quelques cas de gastro-entérites (moins d'une dizaine) signalés en 1985 pour l'ensemble des côtes, les conséquences sanitaires n'ont pas été importantes cette année là. En revanche en 1986, le nombre de personnes intoxiquées est aussi important qu'en 1984 (environ 2 000) et en 1987 c'est également 2000 cas d'intoxication diarrhéique qui sont signalés dans un secteur récemment concerné par ces phénomènes: la côte charentaise. II semble donc, en résumé, que si la mise en place d'un réseau de surveillance a certainement limité le nombre de cas de DSP parmi les consommateurs, il ne permette pas dans l'absolu de se prémunir 
contre des évènements du type : 1) contamination élevée et rapide d'un secteur nouveau et 2) transfert et commercialisation de coquillages contaminés avant la mise en place de l'interdiction de vente.

Sur le plan économique, les durées d'interdiction de vente et le nombre d'établissements d'expédition touchés par ces mesures sont évidemment proportionnels à l'ampleur de la contamination. A titre d'exemple, en 1985 seulement 15 établissements d'expédition ont été fermés contre 432 en 1983,92 en 1984 et 220 en 1986.

\section{b. Gyrodinium aureolum}

On signale des blooms de $G$. aureolum dès 1976 sur les côtes françaises et plus particulièrement à l'extrême ouest de la Bretagne. Ces manifestations sont peu nombreuses (entre 2 et 4 par an entre 1976 et 1988) si l'on ne considère que celles ayant provoqué des mortalités de coquillages et d'autres invertébrés benthiques (fig. 3). On note cependant (fig. 5) une extension vers le nord et le sud des zones touchées par les blooms de Gyrodinium entre 1976 et 1988. Ces observations, néanmoins, sont à considérer avec prudence: si l'exactitude des déterminations de $G$. aureolum dans les secteurs: rade de Brest, baie de Somme ne sont pas à démontrer, il n'en est pas de même au sud de la baie de Vilaine et au nord du Mont St Michel (1986 à 1988) où la diagnose des espèces constituant les "eaux brunes" n'a pas été validée.
Les mortalités de bivalves, crustacés et poissons, les inhibitions de croissance chez Pecten maximus et peut être Ruditapes philippinarum, sont en majorité attribuables à $G$. aureolum depuis 1975 sur le littoral français. De ce fait, les conséquences économiques peuvent être très importantes: en septembre 1980, 50 à 70 $\%$ du cheptel mytilicole a été détruit en baie de Douarnenez, tandis qu'en 1983, 550000 post-larves de $P$. maximus étaient détruites, soit la quasi totalité de la production dans les bassins d'élevage de la rade de Brest.

\section{c. Alexandrium minutum}

Des intoxications PSP ont été - semble-t-il - recensées pour la première fois en France en 1907 à Calais, puis en 1911 à Paris. Dans les deux cas, des décès ont été enregistrés et l'empoisonnement avait reçu l'appelation d'«accident mytilitoxique». Bien que les symptômes s'apparentaient respectivement à ceux du PSP et que les personnes intoxiquées aient consommé des moules, l'agent responsable n'avait pas été identifié.

Entre 1911 et 1988, si l'on excepte l'introduction en 1976 de moules d'Espagne contaminées, aucune intoxication paralytique ne sera recensée sur les côtes françaises et aucune description d'une espèce s'apparentant à Protogonyaulax tamarensis ne pourra être trouvée dans les publications traitant du cycle annuel du phytoplancton en zone littorale. Néanmoins, dès 1984, le réseau de surveillance IFREMER a été conçu de façon à détecter non seulement des 


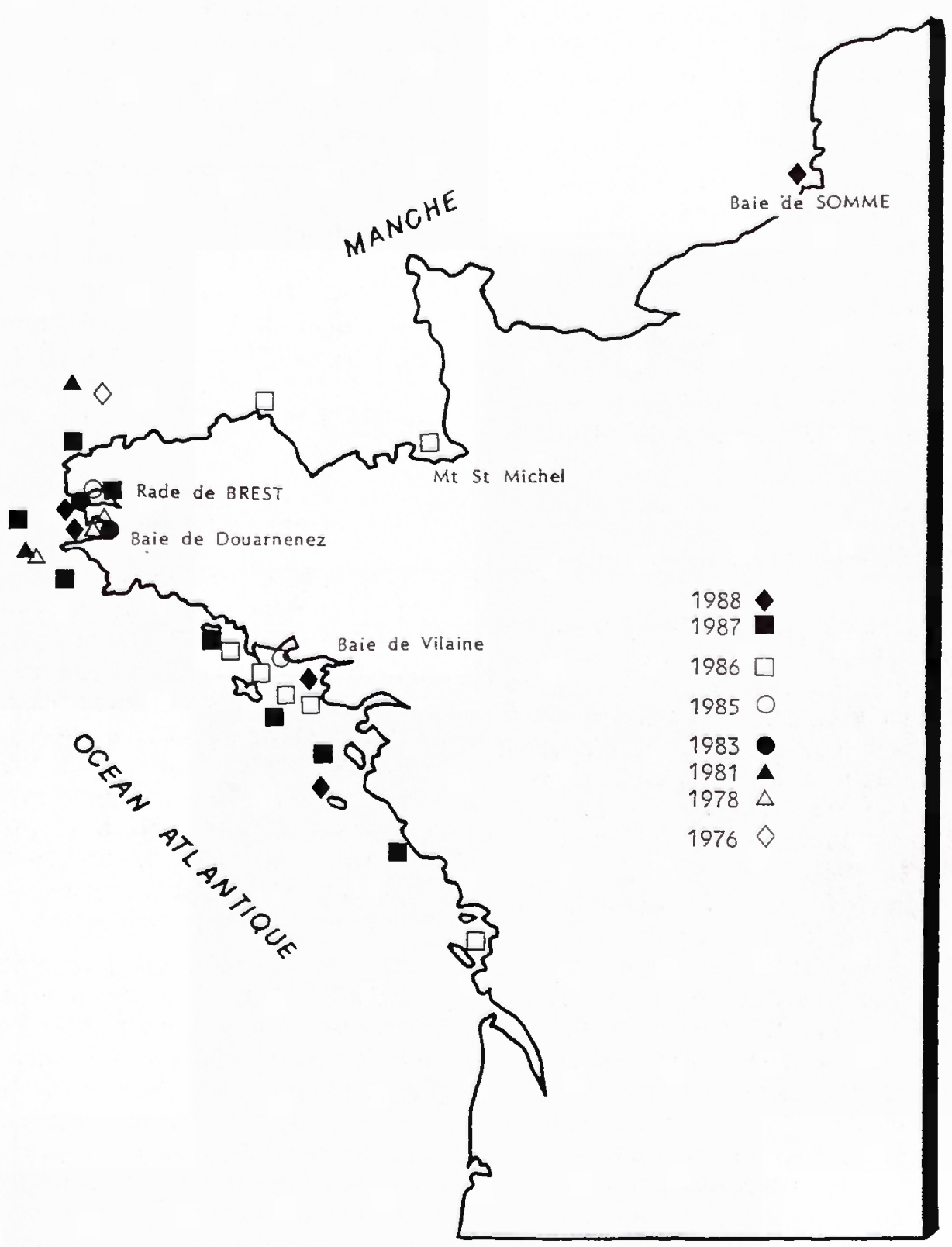

Fig. 5. - Localisation des blooms de G. aureolum le long des côtes françaises de la Manche et de l'Atlantique de 1976 à 1988. Locations of G. aureolum blooms along french Channel and Atlantic coasts from 1976 to 1988. 
a)

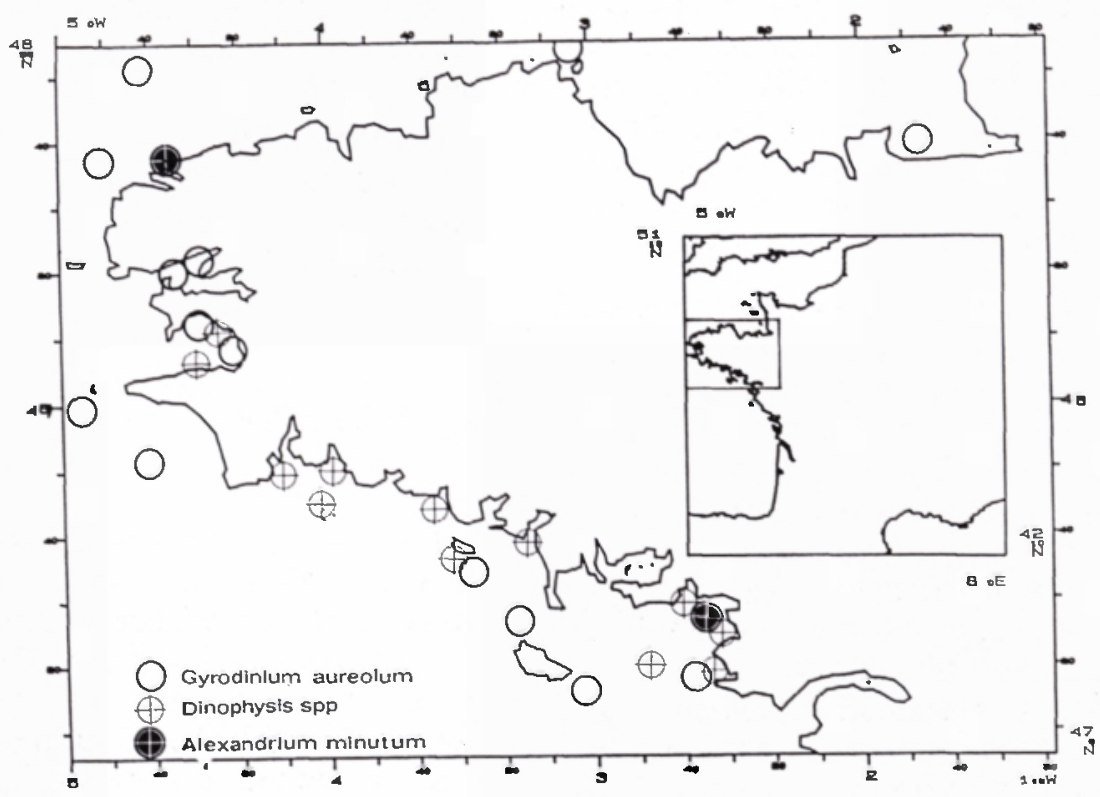

b)

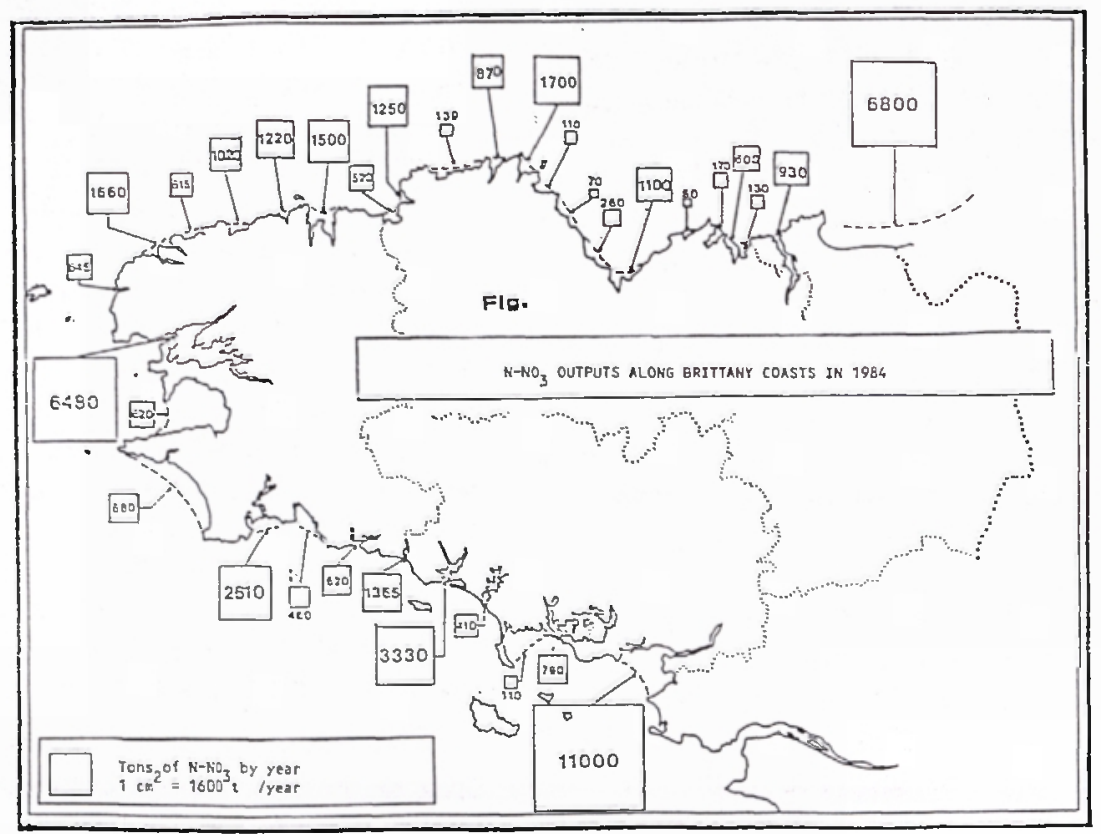

Fig. 6a. - Localisations des blooms de dinoflagellées toxiques en Bretagne Sud entre 1975 et 1988. Locations of toxic dinoflagellates blooms along Brittany coast between 1975 and 1988.

Fig. 6b. - Apports de Nitrates ( $\mathrm{N}-\mathrm{NO}_{3}$ ) sur les côtes Bretonnes en 1984 (Menesguen ef Piriou, 1987). N-NO3 out puts Brittany coasts in 1984. 
concentrations anormales de Dinophysis et Gyrodinium dans l'eau, mais aussi de Protogonyaulax (= Alexandrium $B$ alech), afin de déclancher immédiatement des tests de toxicité sur les coquillages des zones contaminées selon la méthode AOAC.

C'est un scénario de ce type qui a été réalisé en septembre 1988 dans l'Aber Wrach, suite au signalement d'une eau rouge dans la partie amont de ce site. Signalons cependant qu'avant cet évènement plusieurs espèces du type Protogonyaulax avaient été détectées sur nos côtes: Alexandrium minutum en baie de Vilaine (600 000 cellules. $1^{-1}$ ) en juillet 1985 et Goniodoma Pseudogonyaulax (1 200000 cellules. $1^{-1}$ ) dans le bassin d'Arcachon la même année. Par ailleurs, dans la mesure où l'espèce toxique Protogonyaulax tamarensis est présente sur les côtes scandinaves, en Mer du Nord sur la côte est anglaise et sud irlandaise, le risque que nos côtes soient un jour contaminées n'était pas à écarter.

En ce qui concerne l'espèce rencontrée dans l'Aber Wrach, les examens réalisés sur une dissection des plaques thécales ont révélé une morphologie typique de celle d'Alexandrium minutum. Cette espèce s'est révélée récemment toxique $\mathrm{PSP}$ en Australie (HALLEGRAEF et al., 1988) au cours d'eaux rouges en 1986 et 1987 à Adelaide. La toxicité atteinte dans les moules était très élevée: 2 $700 \mu \mathrm{g}$ PSP par $100 \mathrm{~g}$ de chair de bivalve, mais aucun cas d'intoxication n'avait été répertorié du fait de la fermeture de la zone. Bien qu'A. minutum soit très répandu dans le monde, il n'avait jamais été associé à ce type d'intoxication jusqu'à ces deux dernières années.

La contamination d'aôut 1988 en Bretagne nord a entraîné des concentrations maximales de PSP dans les moules de $400 \mu \mathrm{g}$ par $100 \mathrm{~g}$ de chair.

\section{A. DISCUSSION}

II est relativement difficile d'établir clairement s'il y a ou non augmentation évidente du nombre d'eaux colorèes rencensées en 13 ans. On peut néanmoins considérer qu'à partir de 1978 la sensibilisation à ce problème a été importante en France, que ce soit au niveau des organismes de recherche ou de la presse et des médias. De ce fait, même si l'information recueillie à partir de 1978 n'est pas exhaustive, on peut raisonnablement considérer que la "perte d'information" est à peu près constante d'une année à l'autre et que les proportions relatives d'eaux colorées à diatomées et dinoflagellés sont indicatives d'une situation réelle. II en résulte que, si l'on excepte l'année 1978 où un nombre très important d'eaux colorées à été noté, on peut observer une augmentation graduelle du nombre total de cas entre 1979 et 1984 , tandis que la période 1985-1988 est marquée par une régression sensible.

Cependant, si l'on ne considère que le nombre d'eaux colorées à dinoflagellés, il est difficile de déceler une variation significative entre 1981 et 1988. Les zones littorales correspondant aux plus fortes fréquences 
d'eaux colorées pendant ces 13 dernières années sont typiquement la baie de Seine et l'ensemble: baie de Vilaine-baie baie de Quiberon. Ces deux sites sont soumis aux apports de deux estuaires industrialisés de grands fleuves: la Seine et la Loire. En terme de région, c'est la Bretagne sud, avec 71 cas d'eaux colorées en 13 ans qui se révèle être la zone la plus touchée par ces phénomènes.

L'évolution des blooms de dinoflagellés toxiques (qu'ils produisent ou non des eaux colorées) est malheureusement liée à la connaissance des espèces concernées: 1983 pour Dinophysis acuminata et 1988 pour Alexandrium minutum. II paraît donc difficile d'établir avec certitude une quelconque augmentation de leurs apparitions. On peut simplement estimer que les blooms à Gyrodinium aureolum ayant entraîné des mortalités d'animaux marins sont à peu près constants en nombre de 1975 à 1988 , bien qu'apparaissant très irrégulièrement, II est par contre trop tôt pour tenter d'établir, sur une période aussi courte que 1983-1988, si les blooms de Dinophysis présentent bien une augmentation de leur nombre à partir de 1986.

Enfin, et à titre d'exemple, nous avons représenté sur la figure $6 a$ les localisations des blooms à $G$. aureolum. Dinophysis spp et $A$. minutum entre 1975 et 1988 , uniquement pour la Bretagne. Parallèlement, nous avons également représenté sur la figure $6 b$ les apports en Nitrates (en tonnes/an) pour cette même région, en 1984 (MENESGUEN et PIRIOU, 1987), II est certain que les plus forts apports annuels en $\mathrm{N}-\mathrm{NO}_{3}$ (Bretagne sud) coïncident avec la portion de côte la plus touchée par les blooms de dinoflagellés toxiques en 13 ans, Cependant, ce type de généralisation, souvent utilisé par la grande presse, est dangereux. En effet, la côte nord de la Bretagne, moins sujette à des apports importants en nitrates, est néanmoins très touchée par les «marées vertes" à UIva sp., au contraire de la côte sud, Dans ce dernier cas, il s'agit d'une macroalgue reconnue pour utiliser massivement les excès d'azote présents en zone littorale. Pour ce qui est de Gyrodinium aureolum, qui présente également un tropisme certain pour l'azote, il est également curieux de noter que la baie de Douarnenez (620 t/an) est très peu touchée par les apports en $\mathrm{N}-\mathrm{NO}_{3}$ lorsqu'on la compare à d'autres secteurs. Enfin, aucun bloom de Dinophysis n'a pu être corrélé depuis 1984 soit avec l'azote, soit avec le phosphore, que ce soit au large ou dans les baies et les estuaires.

Autrement dit, même si les apports de sels nutritifs ont augmenté ces dix dernières années, il faut se préserver de toute approche subjective.

\section{REFERENCES}

Belin C., Berthome J.P. - 1988 - Bilan des perturbations phytoplanctoniques observées sur les côtes françaises en 1986 - Rapport IFREMER DRV 88008-CSRU/NANTES. $99 \mathrm{p}$.

Berthome J.C., Belin C, - 1988 - Bilan des perturbations phytoplanctoniques observées sur les côtes françaises en 
1985 - Rapport IFREMER DRV 88005-CSRU/NANTES.

Berthome J.C., Lassus P. - 1986 - Bilan de la situation française en matière de contrôle conchylicole en re1ation avec les contaminations par dinoflagellés toxiques. $4^{\theta}$ Colloque franco-japonais. Marseille 1621 sept. 1985 . fasc. 2 : 37 50.

Colebrook J.M., Robinson C.A. , Hunt H.G., Roskell J. et al. - 1984 - Continuous Plankton Records: a possible reversal in the downward trend in the abundance of the plankton of the North Sea and the Northeast Atlantic. J. Cons. int. Explor. Meer, 41 : 304-306.

Hallegraef G.M., Steffensen D.A., Wetherbee, R. - 1988 - Three estuarine Australian dinoflagellates that can produce paralytic Shellfish toxins. Jour. of Plankt. Research. 10 (3) : 533-541.

Lassus P., Maggi P., Bessineton C. - 1980 - Les phénomènes d'eaux colorées de la baie de Seine en 1978. Science et Pêche, Bull. Inst. Pêches Marit., 298 : 1-28.

Lefevre J. - 1979 - On the hypothesis of a relationship between dinoflagellate blooms and the Amoco-Cadiz oil spill. J. Mar. biol. Ass. U.K. 59 : 525-528.

Maddock L., Boalch G.J. and Harbour D.S. - 1981 - populations of phytoplankton in the Western English Channel between 1964 and 1974. J. Mar. biol. Ass. U.K. 61 : 565-583.

Marteil L., Paulmier G. - 1970 - Le phytoplancton des "eaux rouges" sur les côtes européennes de l'Atlantique. ICES CM 1970/L: 13. Comité du plancton.

Menesguen A., Piriou J.Y. - 1987 - Marées vertes à Ulves. in : premières rencontres interrégionales. Recherches appliquées à la gestion des ressources vivantes marines. Arcachon. 12 et 13 février 1988. 9 p.

Radach G., Berg J. - 1986 - Trends in the concentrations of nutrients and phytoplankton in the Helgoland Bight (Helgoland roads data) : Ber. Biol. Anst. Helgol, 63 p. $\mathrm{n}^{\circ} 2$.

Smodlaka N., Revelante N. - 1983 - The trend of phytoplankton production in the northern Adriactic Sea : twelve year survey, Rapp. Comm. Int. Mer. Medi. 28,9 : 89-90. 